\title{
Clinical implementation of chromosomal microarray technology in prenatal diagnosis (Review)
}

\author{
JI UN KANG ${ }^{1}$ and SUN HOE KOO ${ }^{2}$ \\ ${ }^{1}$ Department of Biomedical Laboratory Science, Korea Nazarene University, Cheonan 331-718; \\ ${ }^{2}$ Department of Laboratory Medicine, Chungnam National University College of Medicine, \\ Taejeon 301-721, Republic of Korea
}

Received May 16, 2012; Accepted September 17, 2012

DOI: $10.3892 / \mathrm{mmr} .2012 .1116$

\begin{abstract}
Chromosomal microarray technology represents the technical convergence of molecular genetics and cytogenetics, and is rapidly revolutionizing modern cytogenetics. Expected genomic aberrations are accurately identified and provide readily interpretable results that are suitable for clinical risk stratification and therapeutic strategies. The application of array technology in prenatal genetic diagnosis provides distinct advantages over conventional cytogenetic analysis in detecting both the majority of microscopic and submicroscopic chromosomal abnormalities. In the last few years, the validity of array technology has become obvious to medical and laboratory communities involved in prenatal diagnostic testing. However, whether or not microarray analysis is sufficient for the detection of cytogenetic abnormalities in prenatal diagnosis and if traditional cytogenetics continue to be important in this new era has yet to be confirmed. In the present study, we systematically reviewed the current status of microarray technology in the identification of pathogenic genomic imbalances and discussed practical considerations for its routine implementation in prenatal diagnosis.
\end{abstract}

\section{Contents}

1. Introduction

2. Conventional cytogenetic analysis

3. Chromosomal microarray analysis (CMA)

4. Clinical utility of CMA in prenatal diagnosis

5. Conclusions and final considerations

Correspondence to: Professor Sun Hoe Koo, Chungnam National University Hospital, 640 Daesadong, Jung-Gu, Taejeon 301-721, Republic of Korea

E-mail: shkoo@cnu.ac.kr

Key words: chromosomal microarray technology, cytogenetics, prenatal diagnosis

\section{Introduction}

The development of the bacterial artificial chromosome (BAC) system was partly developed through the Human Genome Project with a view to construct genomic DNA libraries and physical maps for genomic sequencing (1). The presence of BAC clones has become a valuable tool for identifying genomic imbalances in pregnancies to detect chromosomal abnormalities in at-risk fetuses.

The use of this technology has increased successful detection of risk-related abnormalities and provided an alternative for an enhanced level of screening for chromosomal abnormalities in high-risk pregnancies (2). Microarray-detected chromosomal abnormality rates are estimated to range between 5 and $17 \%$ in prenatal diagnosis, compared to normal results obtained from previous routine cytogenetic testing (3).

The implementation of array comparative genomic hybridization $(\mathrm{CGH})$ in postnatal diagnosis has been thoroughly evaluated in the adolescent and adult population, and is now recommended as the first-line diagnostic test for clinically suspected genetic disorders $(4,5)$. However, there are no available concise guidelines establishing the chromosomal microarray analysis (CMA) applications and platforms for a prenatal setting. The controversial question concerns whether or not CMA technology is likely to or should replace the standard karyotype in prenatal diagnostic practice and whether karyotyping and fluorescent in situ hybridization (FISH) remain essential.

In this article, we reviewed the current literature regarding array genomic hybridization in prenatal diagnosis and discussed the benefits and issues regarding the use of microarrays for the prenatal diagnosis of genetic diseases.

\section{Conventional cytogenetic analysis}

Cytogenetic analysis has provided fundamental insight into the molecular pathogenesis of prenatal diagnosis and has been a useful diagnostic tool for the identification of chromosomal abnormalities in at-risk pregnancies (3). Cytogenetic methods including karyotyping, FISH, CGH and multiplex-FISH or spectral karyotyping (SKY) have previously provided valuable diagnostic and prognostic information for the detection of genomic defects in prenatal diagnosis $(6,7)$. Since the 


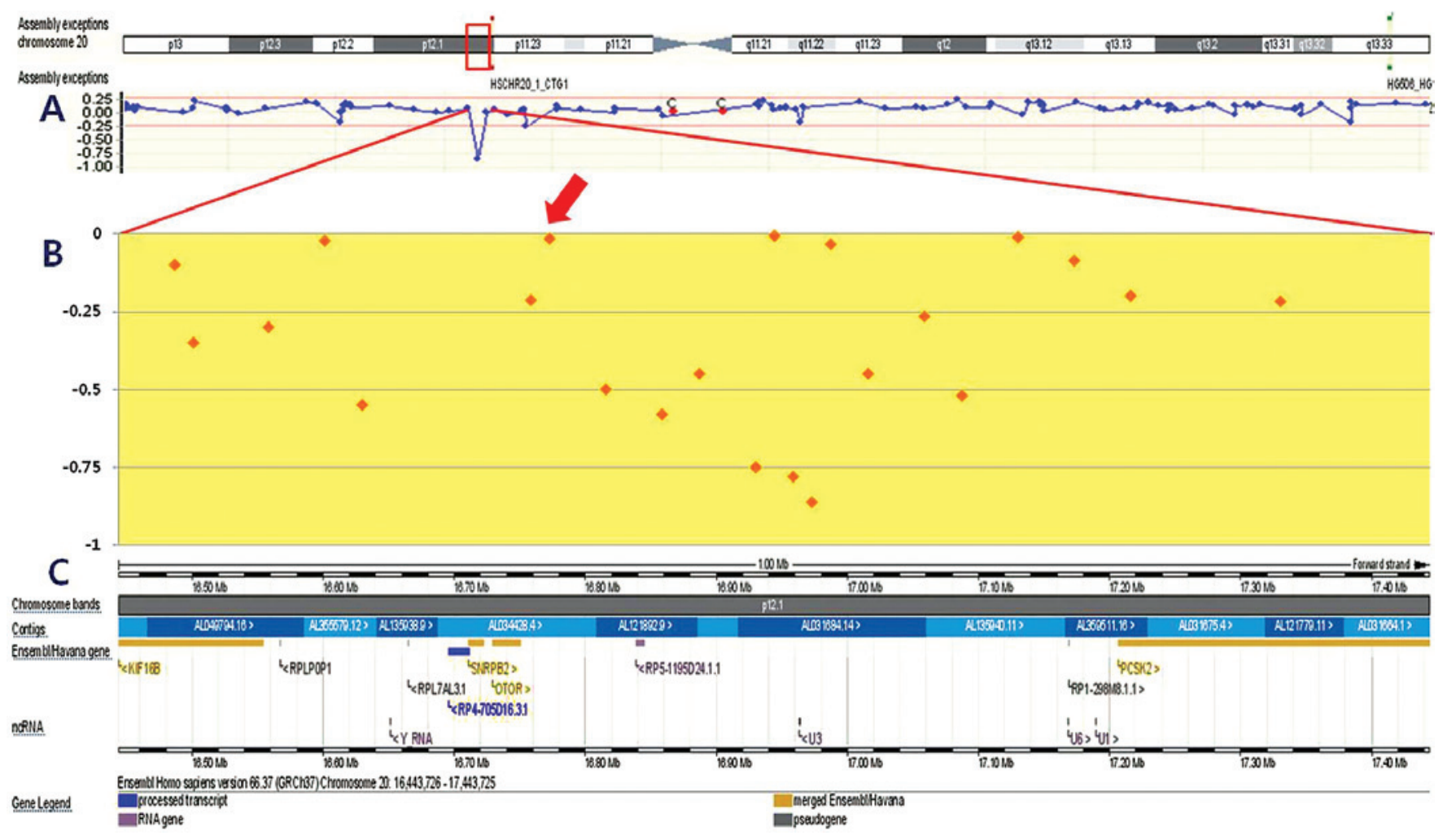

Figure 1. Chromosomal imbalance is detected by array CGH but not by G-banded karyotyping. (A) Increased resolution allowed for the sizing of the segment duplicated at the 20p12.1 region. (B) Arrow points to the close-up view of the duplication at 20p12.1. (C) Genes located within the deleted region (20p12.1) as shown by the UCSC genome browser.

development of chromosome banding techniques in the late 1960s, microscopic analysis has been used as the gold standard for prenatal diagnosis, while in situ hybridization methods have proven to be a useful and reliable technique for identifying and characterizing genomic imbalances. However, these conventional methods have technical limitations, thus resulting in the underestimation of the degree of chromosomal changes. In addition, these methods are also limited by their ability to detect individual DNA screening targets only rather than the entire genome. Furthermore, hidden mosaics, patients with uniparental disomy and complex patterns of meiotic crossing over led to chromosomal aberrations, none of which could be detected by standard cytogenetics or comparative CGH methods. In order to detect such abnormalities, a high-resolution technique is required.

\section{Chromosomal microarray analysis}

CMA circumvents the limitations of conventional cytogenetic techniques. It simultaneously evaluates regions across the entire genome with a higher resolution and an excellent throughput in patients with suspected genome imbalance. This method accurately identifies novel genomic aberrations of possibly uncertain clinical significance not described previously and provides readily interpretable results, suitable for clinical risk stratification and treatment planning (8) (Fig. 1).

The principle behind the array CGH technology is the detection of chromosomal deletions and duplications by comparing equal amounts of genomic DNA from a patient and a healthy control (9). In the array CGH, the two genomes (patient and control) are labeled and co-hybridized onto a glass microscope slide, on which cloned DNA segments have been immobilized (10). The analytic principle involves competition between a differentially-labeled fragmented test and a control diploid DNA, with imbalances due to copy number changes in the test DNA resulting in a shift in the fluorescence spectra (11).

The evaluation is performed by a scanner and the information is then computer-integrated to determine any quantitative deviations in the DNA of the test sample. The primary advantage of CMA is the enhanced detection of copy number anomalies: the deviations that are measurable by molecular means are orders of magnitude smaller than those detectable by light microscopy (12). Common protocols for the application and interpretation of genomic arrays in prenatal diagnosis are capable of decreasing the risk of unexpected findings.

\section{Clinical utility of CMA in prenatal diagnosis}

Array technology is rapidly taking over cytogenetic laboratories, resulting in ability for greatly improved visualization and validation. The increased diagnostic potential of the microarray has naturally led to the need for its use in the prenatal setting. In recent years, the application of microarray-based genomic copy number analysis has proven to be beneficial, allowing for proper counseling and providing the parents with all the tools for a conscious decision regarding their pregnancy.

There are several studies available aiming to assess the diagnostic ability of array CGH in the screening of hidden chromosomal aberrations in prenatal genetic diseases with an apparently normal karyotype (13-15). Depending on the 
ascertainment criteria and the level of resolution achieved in the cytogenetic assessment, the microarray prevails in the detection of copy number anomalies, by identifying pathogenic abnormalities in up to $16 \%$ of fetuses with an abnormal ultrasound and normal karyotype (13).

In the study by Vialard et al (14), array CGH diagnosed two de novo unbalanced karyotypes and four additional abnormalities that could not be identified with conventional cytogenetic methods in classic microdeletion syndromes and subtelomeric rearrangements in 39 fetuses with multiple congenital abnormalities after the pregnancy was terminated. A previous analysis of eight prenatal studies using the array technology from various platforms also concluded that array CGH detected a $3.6 \%$ increase in genomic imbalances when conventional karyotyping was normal, regardless of the indication for referral. When the referral indication was abnormal in the ultrasound, this percentage increased to $5.2 \%$ (13).

More recently, a Canadian study using array $\mathrm{CGH}$, has demonstrated the identification of an additional $8.2 \%$ of positive diagnosis in 49 fetuses with major malformations that were not visible with karyotyping (12). In the experiment of Le Caignec et al, (16) the array platform detected all cytogenetic abnormalities previously analyzed by $\mathrm{G}$ banding and revealed new rearrangements in $7-10 \%$ of the cases from chorionic villus culture in 41 products of conception. Emerging data from D'Amours et al (17) also reported a relatively high percentage of findings of unclear clinical significance in $12.2 \%$ of the tested fetuses. These observations demonstrated that the potential of the array $\mathrm{CGH}$ to reveal the cryptic and/ or complex nature of chromosome arrangements otherwise undetectable by chromosome analysis markedly increases the elucidation of prenatal genetic diseases.

Additional cases have also emphasized the importance of further investigation on microarray technology since other imbalances underlying more serious consequences may be present. Maitz et al (18) reported a characteristic case concerning a 21-week gestation fetus with a complex congenital heart defect and no other ultrasound abnormalities. The karyotype carried out by conventional cytogenetic analysis was normal. FISH analysis by the Di George/VCFS probe, combined with a control probe mapping to the $22 \mathrm{q} 13.3$ region (ARSA) was performed, excluding the 22q11.2 deletion and showing only one signal from the ARSA locus. Microarray analysis demonstrated that a $6.5 \mathrm{Mb}$ interstitial deletion was in fact present at 22q13.3, leading to hemizygosity in several genes (19). Findings of a similar study by Wat et al (20) also demonstrated that the high frequency of cardiac and diaphragmatic defects associated with 8p23.1 interstitial deletions that were detected by microarray analysis were not identified by conventional chromosome analysis. These findings prove that in isolated ultrasound heart abnormalities and a normal karyotype, FISH analysis is not adequate, and should therefore be substituted by microarray analysis.

Considering the advantages and the lack of additional risk of array $\mathrm{CGH}$ for the patients, it is reasonable to suggest that this test be offered to all women already undergoing invasive testing (21). In the study conducted by Van den Veyver et al (2), only 4 (22\%) of 18 abnormal prenatal array CGH cases had abnormal ultrasound findings as the sole indication for testing, suggesting that testing should not be limited to pregnancies with known abnormal ultrasound findings. Wat et al (20) also suggested that array $\mathrm{CGH}$ be performed on all prenatal cases with congenital cardiac and/or diaphragm defects. When offered to choose between karyotyping and array CGH, 74\% of the parents chose the latter method (21).

Another crucial instance requiring the application of microarray is the presence of a de novo reciprocal translocation or a de novo supernumerary marker chromosome in the fetal karyotype (22). Previous studies (23-25) demonstrated that cryptic deletions are present either at the translocation breakpoints or elsewhere in the genome in approximately $40 \%$ of the de novo reciprocal translocations detected in patients with phenotypic abnormalities, explaining the phenotypegenotype correlation. Since the breakpoints of the great majority of reciprocal translocations are non-recurrent, it is obvious that only the array platforms covering most of the genome have the potential to detect deletions associated with reciprocal translocations.

A high-resolution array platform covering the entire genome would therefore provide much more informative results than one containing only low coverage limited to prenatal disease-associated regions. Although microarray technology does not have the potential to detect polyploidy and balanced chromosomal rearrangements, these are relatively infrequent causes of abnormal phenotypes in a typical referral population. The frequency of pathogenic de novo reciprocal translocations due to the breakage of a dosage-sensitive gene or its long-range controlling region is extremely low (22), and polyploidy is almost always lethal during fetal life and is generally detected on ultrasound investigation (26). In case of such a suspicion, conventional karyotyping detects balanced chromosomal rearrangements. The majority of truly balanced translocations generate no phenotypic abnormality (27) and their identification leads to difficult clinical decisions during pregnancy. The American College of Obstetricians and Gynecologists (ACOG) (28) suggested that conventional karyotyping remains the principal tool for prenatal diagnosis and targeted arrays be offered as an adjunct in cases with abnormal prenatal anatomical findings and a normal conventional karyotype (21).

Given the potential described in this review, we anticipate the array $\mathrm{CGH}$ to be the initial prenatal diagnostic approach for the identification of chromosomal abnormalities in the near future. Although clinical application of array $\mathrm{CGH}$ as a universal routine test for genetic diagnosis is premature, further investigation may allow for an evaluation of the overall diagnostic yield of microarray technology over routine prenatal testing with conventional karyotype, as well as cost effectiveness (29).

\section{Conclusions and final considerations}

In this review, we presented the potential utility of array CGH for the detection of chromosomal abnormalities in prenatal diagnosis. This new platform, with its potential to decrease the risk of unexpected findings, is likely to be the first-line test for detecting chromosomal abnormalities in prenatal diagnosis.

In order to reach a consensus regarding the optimum configuration of an array, additional investigations carried out on large-scale populations that have undergone both karyotyping and a commercially reproducible array are required. 


\section{Acknowledgements}

This study was financed by the research fund of the Korea Nazarene University in 2012.

\section{References}

1. De Braekeleer E, Douet-Guilbert N, Basinko A, et al: Using bacterial artificial chromosomes in leukemia research: the experience at the university cytogenetics laboratory in Brest, France. J Biomed Biotechnol: 329471, 2011.

2. Van den Veyver IB, Patel A, Shaw CA, et al: Clinical use of array comparative genomic hybridization $(\mathrm{aCGH})$ for prenatal diagnosis in 300 cases. Prenat Diagn 29: 29-39, 2009.

3. Lee CN, Lin SY, Lin CH, Shih JC, Lin TH and Su YN: Clinical utility of array comparative genomic hybridisation for prenatal diagnosis: a cohort study of 3171 pregnancies. BJOG: Feb.07, 2012 (E-pub ahead of print).

4. Shen Y, Dies KA, Holm IA, et al: Clinical genetic testing for patients with autism spectrum disorders. Pediatrics 125: 727-735, 2010.

5. Miller DT, Adam MP, Aradhya S, et al: Consensus statement: Chromosomal microarray is a first-tier clinical diagnostic test for individuals with developmental disabilities or congenital anomalies. Am J Hum Genet 86: 749-764, 2010.

6. Kang JU, Koo SH, Kwon KC, Park JW, Shin SY, Kim JM and Jung SS: High frequency of genetic alterations in non-small cell lung cancer detected by multi-target fluorescence in situ hybridization. J Korean Med Sci 22: S47-S51, 2007.

7. Kang JU, Koo SH, Jeong TE, Kwon KC, Park JW and Jeon CH: Multitarget fluorescence in situ hybridization and melanoma antigen genes analysis in primary bladder carcinoma. Cancer Genet Cytogenet 164: 32-38, 2006.

8. Kang JU, Koo SH, Kwon KC and Park JW: Frequent silence of chromosome 9p, homozygous DOCK8, DMRT1 and DMRT3 deletion at 9p24.3 in squamous cell carcinoma of the lung. Int J Oncol 37: 327-335, 2010

9. Kang JU, Koo SH, Kwon KC, Park JW and Kim JM: Identification of novel candidate target genes, including EPHB3, MASP1 and SST at 3q26.2-q29 in squamous cell carcinoma of the lung. BMC Cancer 9: 237, 2009.

10. Shaffer LG and Bejjani BA: Medical applications of array $\mathrm{CGH}$ and the transformation of clinical cytogenetics. Cytogenet Genome Res 115: 303-309, 2006.

11. Maciejewski JP and Mufti GJ: Whole genome scanning as a cytogenetic tool in hematologic malignancies. Blood 15: 965-974 2008.

12. Duncan A and Langlois S: SOGC Genetics Committee: CCMG Prenatal Diagnosis Committee.Use of array genomic hybridization technology in prenatal diagnosis in Canada. J Obstet Gynaecol Can 33: 1256-1259, 2011.

13. de Ravel TJ, Devriendt K, Fryns JP and Vermeesch JR: What's new in karyotyping? The move towards array comparative genomic hybridisation (CGH). Eur J Pediatr 166: 637-643, 2007.

14. Vialard F, Molina Gomes D, Leroy B, et al: Array comparative genomic hybridization in prenatal diagnosis: another experience. Fetal Diagn Ther 25: 277-284, 2009.

15. Hillman SC, Pretlove S, Coomarasamy A, et al: Additional information from array comparative genomic hybridization technology over conventional karyotyping in prenatal diagnosis: a systematic review and meta-analysis. Ultrasound Obstet Gynecol 37: 6-14, 2011.
16. Le Caignec C, Boceno M, Saugier-Veber P, Jacquemont S, Joubert M, David A, Frebourg T and Rival JM: Detection of genomic imbalances by array based comparative genomic hybridisation in fetuses with multiple malformations. J Med Genet 42: 121-128, 2005.

17. D'Amours G, Kibar Z, Mathonnet G, Fetni R, Tihy F, Désilets V, Nizard S, Michaud JL and Lemyre E: Whole-genome array CGH identifies pathogenic copy number variations in fetuses with major malformations and a normal karyotype. Clin Genet 81: 128-141, 2012.

18. Maitz S, Gentilin B, Colli AM, et al: Expanding the phenotype of 22q13.3 deletion: report of a case detected prenatally. Prenat Diagn 28: 978-980, 2008

19. Zuffardi O, Vetro A, Brady P and Vermeesch J: Array technology in prenatal diagnosis. Semin Fetal Neonatal Med 16: 94-98, 2011.

20. Wat MJ, Shchelochkov OA, Holder AM, et al: Chromosome 8p23.1 deletions as a cause of complex congenital heart defects and diaphragmatic hernia. Am J Med Genet A 149A: 1661-1677, 2009.

21. Lichtenbelt KD, Knoers NV and Schuring-Blom GH: From karyotyping to array-CGH in prenatal diagnosis. Cytogenet Genome Res 135: 241-250, 2011.

22. Breman AM, Bi WM and Cheung SW: Prenatal diagnosis by array-based comparative genomic hybridization in the clinical laboratory setting. Beijing Da Xue Xue Bao 18: 500-504, 2009.

23. De Gregori M, Ciccone R, Magini P, et al: Cryptic deletions are a common finding in 'balanced' reciprocal and complex chromosome rearrangements: a study of 59 patients. J Med Genet 44: 750-762, 2007.

24. Baptista J, Mercer C, Prigmore E, et al: Breakpoint mapping and array $\mathrm{CGH}$ in translocations: comparison of a phenotypically normal and an abnormal cohort. Am J Hum Genet 82: 927-936, 2008.

25. Schluth-Bolard C, Delobel B, Sanlaville D, et al: Cryptic genomic imbalances in de novo and inherited apparently balanced chromosomal rearrangements: array $\mathrm{CGH}$ study of 47 unrelated cases. Eur J Med Genet 52: 291-296, 2009.

26. Cain CC, Saul DO, Oehler E, Blakemore K and Stetten G: Prenatal detection of a subtle unbalanced chromosome rearrangement by karyotyping, FISH and array comparative genomic hybridization. Fetal Diagn Ther 24: 286-290, 2008.

27. Warburton D: De novo balanced chromosome rearrangements and extra marker chromosomes identified at prenatal diagnosis: clinical significance and distribution of breakpoints. Am J Hum Genet 49: 995-1013, 1991.

28. ACOG Committee No. 446: Array comparative genomic hybridization in prenatal diagnosis. Obstet Gynecol 114: 1161-1163, 2009.

29. Evangelidou P, Sismani C, Ioannides M, Christodoulou C, Koumbaris G, Kallikas I, Georgiou I, Velissariou V and Patsalis PC: Clinical application of whole-genome array CGH during prenatal diagnosis: Study of 25 selected pregnancies with abnormal ultrasound findings or apparently balanced structural aberrations. Mol Cytogenet 3: 24, 2010. 